\title{
Crop germplasm: Molecular and physiological perspective towards achieving global crop sustainability
}

\author{
Raju Mondal $^{1 *}$ and Amit $\mathrm{Kumar}^{2 *}$ \\ ${ }^{1}$ Mulberry Tissue Culture Lab, Central Sericultural Germplasm Resources Centre (CSGRC), \\ Ministry of Textile, Govt. of India, Hosur - 635109, India \\ ${ }^{2}$ Host Plant Section, Central Muga Eri Research \& Training Institute, Central Silk Board, \\ Ministry of Textile, Govt. of India, Lahdoigarh, Jorhat, Assam-785700, India
}

*To be considered as the joint corresponding author

*Email: Raju Mondal, rmcrijaf@yahoo.in,https://orcid.org0000-0001-7526-1940

*Email: Amit Kumar, amit_bio80@yahoo.com, https://orcid.org/0000-0003-1956-0174

Keyword: Artificial intelligence, Core-set, Climate change, Nitrogen use efficiency (NUE), Omic approaches. Plant genetic resources, Stress, System biology, Water use efficiency (WUE) 


\title{
Crop germplasm: Molecular and physiological perspective for achieving global crop sustainability
}

\begin{abstract}
:
Germplasm is a long-term resource management mission and investment for civilization. For both food and nutritional health, the present changing environmental scenario has become an urgent universal concern. Multiple excellent studies have been previously performed, although the advancement and innovation of practices will require the exploration of the potentiality of crop germplasm. In this study, we emphasized (i) germplasm activates, current challenges and ongoing trends of the crop germplasm, and (ii) how the system biology will be helpful to understand the complex traits such as water use efficiency (WUE), and nitrogen use efficiency (NUE) to mitigate challenges for sustainable development under growing food requirement and climate change conditions. We focused on a vision for transforming PGR into a bio-digital resource system, for the development of climate-smart crops for sustainable food production. Moreover, this review attempted to address current challenges, research gaps and describe the advanced integrated strategies that could provide a platform for future crop improvement research.
\end{abstract}

Keyword: Artificial intelligence, Core set, Climate change, Nitrogen use efficiency, Omic approaches, Plant genetic resources, Stress, Systems biology, Water use efficiency

\section{Introduction}

By introducing the Rht (reduced height) gene, the 60-year-old global success stories of the first green revolution (1960s) was achieved. Subsequently, the importance of crop germplasm and the conservation of crops have been recognized worldwide. To date, an estimated $\sim 1750$ plant germplasm centres globally hold 7.4 million accessions; however, $<2 \%$ of these assets have been used as plant genetic resources (PGRs) (Janzen et al., 2019). Recently, statistics suggested that the existing crop yield trajectory is insufficient to nourish the global population in 2050 (Bailey-Serres et al., 2019). Moreover, by 2050, the global food demand will be expected to increase by $70 \%$ (Connor et al. 2017). Many decades later, because of global food security and increasing climate-related challenges, geneticists realized an urgent requirement for advanced approaches to comprehensively assess PGRs for potential use. Faced with these post-green revolution challenges, emerging crop breeding techniques should attempt to reach increasing requirements at a reasonable rate of crop growth. At present, the major challenge is how to exploit and capitalize on this abundant resource. In this 
circumstance, for future life insurance, a vision has been proposed for transforming PGRs into a bio-digital resource system. To harness the benefit of PGRs, despite well-equipped maintenance and storage facilities, the additional execution of frontier technology transfer is lacking.

\section{Germplasm activity, trends and current challenges}

The breeding resource maintenance program is the first important component of digging into the PGRs. Figure 1 shows the structural component and activity of germplasm are divided into four major components. To harvest the benefit of PGRs, the development and maintenance of a precise "core-set" collection has been illustrated. The core-set collections should have the greatest diversity of total genetic resources (comprising both exotic and endogenous collections) because they served as a reference population for additional breeding program (Mascher et al., 2019). The core-set collection should be free of genetic duplicates and must have evolutionary potential genotypes. Basic cytogenetic information such as ploidy level, mitotic behaviour, and genetic diversity/redundancy is required for an additional breakthrough. Such a core-set will be useful to understand the genetic variation in the species that can be used for innovative field experiments and subsequent parental material in the breeding program. Moreover, the cost of maintenance of germplasm could be also be reduced. 


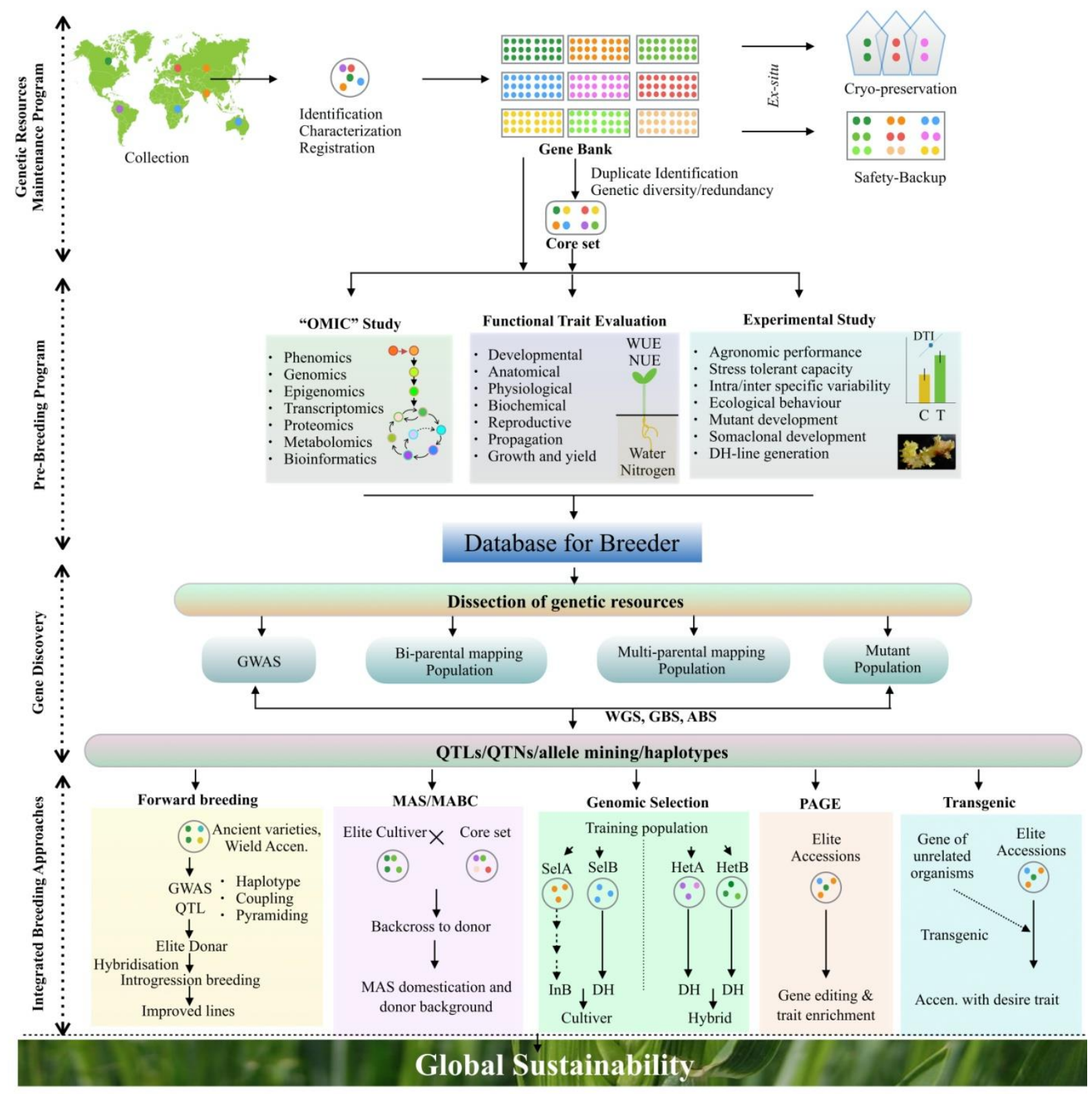

Figure 1: Structure and activity of crop improvement strategies for exploration and capitalization of genetic resources. Concerned crop improvement program divided into four major strategies. (A) Genetic Resource Maintenance Program: A collective model for collection to conserving breeding resources. Integrated taxonomic and cytogenetic approaches have been proposed for developing the "core-set" germplasm. A core-set collection represents a reference population of total germplasm with unique accessions. (B) Pre-Breeding Program: Advance pre-breeding strategy has been emphasized to reveal the potentiality of the core collection. Integrated omicapproaches, functional teat evaluation, and different experimental datasets can be generating a database for plant geneticists/breeders. (C) Gene Discovery: For both complex traits and simple inheritance traits, the most challenging job is to harness the beneficial gene dissecting. GWAS, bi/multi-parental, and 
mutation population mapping are preferably powerful strategies for establishing genetic trait association. Phenome QTL (phQTL), metabolome QTL (mQTL), proteome QTL (pQTL) and expression QTL (eQTL) have been extensively used to discover genes and functional annotation. (D) Integrated Breeding Approaches: Forward breeding, MAS/MABC, PAGE, and transgenic approach can be employed to integrate the simply inherited trains in which genomic selection can be employed to integrate the complex inherited trains. The aim of global sustainability will be fulfilled by this systematic evaluation of genetic resources that will simultaneously save time and budget expenditure. Abbreviation: WUE, water use efficiency; NUE, nitrogen use efficiency; DTI, drought stress index=(T/C)*100; C, control; T, treatment; GWAS, genome-wide association study; QTL, quantitative trait locus; QTN, quantitative trait nucleotide; WGS, whole-genome sequencing; GBS, genotyping by sequencing; ABS, array-based sequencing; MAS, marker-assisted selection; MABC, markerassisted backcrossing; PAGE, promotion of alleles through genome editing; SelA/B, selfing line A/B; HetA/B, heterozygous line A/B; InB, Inbred; DH, doubled haploid.

In the context to explore genetic resources, (1) sink of genetic diversity with increase in the number of gene bank accessions; (2) accumulation of duplicate accessions over a longer period collection; (3) degradation of collection, particularly exotic accessions in response to the edaphic/biotic-abiotic factors; (4) loss of originality because of long-term domestication; (5) insufficient knowledge for characterizing complex important treats; (6) accumulation of rare deleterious alleles during crop domestication; and (7) low recombination frequency between the wild relatives have been considered the major bottleneck for crop improvement (Langridge et al., 2019). Hence, clearly, new and innovative utilization strategies are required to reduce the complexity of these limitations. Therefore, for crop improvement, it is important to intelligently guide the germplasm resources. Accordingly, we should focus on a vision for transforming PGRs into a bio-digital resources system.

Currently, systems biology is considered as a potential approach for the complete understanding of biological systems (Lavarenne et al., 2018). Integrated phenomics and nextgeneration sequencing (NGS) technologies have been utilized for identifying genetic markers associated with desirable traits in multiple crops, including rice, foxtail millet, pigeon pea, pearl millet, cotton, rapeseed, chickpea, and grape. Moreover, the large-scale re-sequencing 
of germplasm accessions have been re-sequenced in rice (3010), pearl millet (994), and chickpea (429) with the ongoing and deep profound of sequencing costs (Varshney et al., 2020). This study suggests that large-scale sequencing should be undertaken for all available accessions. Such projects will generate 'big data' that has storage and computational challenges. Therefore, the advancement of sequence-based study in next-generation breeding, high-throughput bioinformatics platforms, and passionate scientists is required. These datasets will provide the information to breeders for mining superior alleles/haplotypes that can serve as the key to select precious parental material for breeding.

Breeders mostly target functional traits, such as water use efficiency (WUE) and nitrogen use efficiency (NUE), to improve climate resilience crop productivity (Tracy et al, 2020). Agriculture is the world's largest consumer of water and accounts for $70 \%$ of all water used worldwide; by 2050, global food demand is expected to increase by $70 \%$ (Connor et al. 2017). The ongoing global catastrophe of water is one of the major problems in the presentday climate scenarios and drought is considered as the most severe abiotic stress affecting crop productivity at a global level. Moreover, nitrogen is the most essential nutrient for plant's growth, development, and yield. Increasing trends of fertilizer application to achieving productivity, resulting in the decreasing the acquisition and subsequent utilization of applied nitrogen in crop plants. Additionally, the surplus amounts of inorganic and organic nitrogen fertilizers often imposed drastic negative impacts on the environment (Kumar et al., 2020). For example, NUE for cereal production is $\sim 33 \%$ globally and the unaccountable $67 \%$ corresponds to a $\$ 15.9$ billion loss/year of nitrogen fertilizer (Raun et al., 1999; Fagodiya et al., 2020). Moreover, it is estimated that $\sim 1 \%$ increase in crop NUE could annually save $\$ 1.1$ billion (Kant et al., 2011; Li et al., 2020). Hence, for achieving greater agricultural sustainability, the development of crop plants with drought-tolerant and more efficient nitrogen usage is, therefore, an important research challenge. Unfortunately, to date, extremely few studies has been conducted (Table 1). 
Table 1: List of important original research on different crops and detailed information about complex traits - water use efficiency (WUE) and nitrogen use efficiency (NUE)

\begin{tabular}{|c|c|c|c|c|c|c|c|c|}
\hline $\begin{array}{c}\text { Complex } \\
\text { traits }\end{array}$ & $\begin{array}{l}\text { Studied } \\
\text { Crop }\end{array}$ & Species & $\begin{array}{l}\text { Number of } \\
\text { Accession/line } \\
\text { s studied }\end{array}$ & $\begin{array}{c}\text { Condition } \\
\text { of } \\
\text { experiment }\end{array}$ & $\begin{array}{c}\text { Associate } \\
\text { traits studied }\end{array}$ & $\begin{array}{c}\text { Approaches } \\
\text { used }\end{array}$ & Identified gene/functions & $\begin{array}{c}\text { Referenc } \\
\text { es }\end{array}$ \\
\hline \multicolumn{9}{|l|}{ WUE } \\
\hline & Apple & $\begin{array}{l}\text { Malus } \\
\text { domestica }\end{array}$ & $\begin{array}{l}\text { Two } \\
\text { MdATG8i- } \\
\text { overexpressing } \\
\text { lines }\end{array}$ & $\begin{array}{l}\text { Long-term } \\
\text { drought } \\
\text { treatment }\end{array}$ & $\begin{array}{l}\text { Autophagic } \\
\text { activity }\end{array}$ & Transgenic & $\begin{array}{l}\text { MdATG8i-overexpressed } \\
\text { lines exhibited higher WUE } \\
\text { under long-term moderate } \\
\text { drought conditions. }\end{array}$ & $\begin{array}{l}\text { Jia et al., } \\
2021\end{array}$ \\
\hline & Sorghum & $\begin{array}{l}\text { Sorghum } \\
\text { bicolor }\end{array}$ & 659 accessions & $\begin{array}{l}\text { Decrease } \\
\text { light } \\
\text { condition }\end{array}$ & $\begin{array}{l}\text { Stomatal } \\
\text { responses }\end{array}$ & $\begin{array}{l}\text { Genomics, } \\
\text { transcriptomics, } \\
\text { and phenomics } \\
\text { (High- } \\
\text { throughput } \\
\text { thermal } \\
\text { imaging) }\end{array}$ & $\begin{array}{l}\text { Total } 77 \text { genes related to } \\
\text { WUE were identified. Out } \\
\text { of those, } 24 \text { genes involved } \\
\text { in stomatal opening/closing, } \\
35 \text { genes stomatal/epidermal } \\
\text { cell development, } 12 \text { genes } \\
\text { leaf/vasculature } \\
\text { development, } 8 \text { genes- } \\
\text { chlorophyll } \\
\text { metabolism/photosynthesis. }\end{array}$ & $\begin{array}{l}\text { Pignon et } \\
\text { al., } 2021\end{array}$ \\
\hline & Sorghum & $\begin{array}{l}\text { Sorghum } \\
\text { sp. }\end{array}$ & 18 accessions & $\begin{array}{l}\text { Under } \\
\text { fluctuating } \\
\text { light are }\end{array}$ & -- & $\begin{array}{l}\text { Performance- } \\
\text { based on } \\
\text { phenotyping }\end{array}$ & -- & $\begin{array}{l}\text { Pignon et } \\
\text { al., } 2021\end{array}$ \\
\hline & Cotton & $\begin{array}{l}\text { Gossypium } \\
\text { spp. }\end{array}$ & $\begin{array}{l}\text { Two } \\
\text { accessions and } \\
\text { eight cultivars }\end{array}$ & $\begin{array}{l}\text { Controlled } \\
\text { condition }\end{array}$ & Root traits & $\begin{array}{l}\text { Performance- } \\
\text { based on } \\
\text { phenotyping }\end{array}$ & -- & $\begin{array}{l}\text { St Aime } \\
\text { et al., } \\
2021\end{array}$ \\
\hline & $\begin{array}{l}\text { Persian } \\
\text { walnut }\end{array}$ & $\begin{array}{l}\text { Juglans } \\
\text { regia }\end{array}$ & $\begin{array}{l}95 \text { walnut } \\
\text { families }\end{array}$ & $\begin{array}{l}\text { Well- } \\
\text { watered } \\
\text { (control) } \\
\text { and drought }\end{array}$ & Leaf-traits & $\begin{array}{l}\text { Genomics and } \\
\text { performance- } \\
\text { based on } \\
\text { phenotyping }\end{array}$ & $\begin{array}{l}\text { WUE traits were involved } \\
\text { in ABA signaling. }\end{array}$ & $\begin{array}{l}\text { Arab et } \\
\text { al., } 2020\end{array}$ \\
\hline
\end{tabular}


stress

\begin{tabular}{|c|c|c|c|c|c|c|c|}
\hline & & & stress & & & & \\
\hline Cassava & $\begin{array}{l}\text { Manihot } \\
\text { esculenta }\end{array}$ & $\begin{array}{l}\text { High-yielding } \\
13 \text { farm- } \\
\text { preferred } \\
\text { African } \\
\text { cultivars }\end{array}$ & $\begin{array}{l}\text { Controlled } \\
\text { condition }\end{array}$ & $\begin{array}{l}\text { Photosyntheti } \\
\text { c traits }\end{array}$ & $\begin{array}{l}\text { Biochemical } \\
\text { and } \\
\text { performance- } \\
\text { based on } \\
\text { phenotyping }\end{array}$ & -- & $\begin{array}{l}\text { De Souza } \\
\text { et al., } \\
2020\end{array}$ \\
\hline Rice & $\begin{array}{l}\text { Oryza } \\
\text { sativa } L .\end{array}$ & 173 accession & $\begin{array}{l}\text { Water- } \\
\text { limited }\end{array}$ & Root traits & $\begin{array}{l}\text { Marker-trait } \\
\text { associations } \\
\text { (MTAs) }\end{array}$ & -- & $\begin{array}{l}\text { Raju et } \\
\text { al., } 2016\end{array}$ \\
\hline Rice & $\begin{array}{l}\text { Oryza } \\
\text { sativa } L .\end{array}$ & $\begin{array}{l}260 \\
\text { introgressed } \\
\text { lines of IR-64 }\end{array}$ & $\begin{array}{l}\text { water- } \\
\text { limited }\end{array}$ & Root traits & $\begin{array}{l}\text { Performance- } \\
\text { based on } \\
\text { phenotyping }\end{array}$ & -- & $\begin{array}{l}\text { Dharmap } \\
\text { pa et al., } \\
2019\end{array}$ \\
\hline Soybean & $\begin{array}{l}\text { Glycine } \\
\max (\text { L. }) \\
\text { Merr. }\end{array}$ & 10 genotype & $\begin{array}{l}\text { Irrigation } \\
\text { and no- } \\
\text { irrigation }\end{array}$ & $\begin{array}{l}\text { Yield, and } \\
\text { root traits }\end{array}$ & $\begin{array}{l}\text { Performance- } \\
\text { based on } \\
\text { phenotyping }\end{array}$ & -- & $\begin{array}{l}\text { Fried et } \\
\text { al., } 2019\end{array}$ \\
\hline Wheat & - & 71 accessions & $\begin{array}{l}\text { Water- } \\
\text { limited }\end{array}$ & $\begin{array}{l}\text { Different } \\
\text { yield-related } \\
\text { parameters }\end{array}$ & $\begin{array}{l}\text { Performance- } \\
\text { based on } \\
\text { phenotyping }\end{array}$ & -- & $\begin{array}{l}\text { Meena et } \\
\text { al., } 2019\end{array}$ \\
\hline Soybean & $\begin{array}{l}\text { Glycine } \\
\max L . \\
\text { Merr }\end{array}$ & $\begin{array}{l}383 \text { soybean } \\
\text { recombinant } \\
\text { inbred lines }\end{array}$ & $\begin{array}{l}\text { Induced } \\
\text { drought }\end{array}$ & grain yield & $\begin{array}{l}\text { Genome-wide } \\
\text { association } \\
\text { analysis }\end{array}$ & -- & $\begin{array}{l}\text { Lopez et } \\
\text { al, } 2019\end{array}$ \\
\hline Wheat & $\begin{array}{l}\text { Triticum } \\
\text { aestivum L. }\end{array}$ & 100 accessions & INA & $\begin{array}{l}\text { Canopy } \\
\text { temperature, } \\
\text { chlorophyll } \\
\text { content, and } \\
\text { spot blotch } \\
\text { (Cochliobolus } \\
\text { sativus) }\end{array}$ & $\begin{array}{l}\text { Performance- } \\
\text { based on } \\
\text { phenotyping }\end{array}$ & -- & $\begin{array}{l}\text { Jamil et } \\
\text { al., } 2016\end{array}$ \\
\hline
\end{tabular}

sativus) African 


\begin{tabular}{|c|c|c|c|c|c|c|c|c|}
\hline \multicolumn{9}{|l|}{ NUE } \\
\hline & $\begin{array}{l}\text { Rice, } \\
\text { Wheat, } \\
\text { and } \\
\text { Barley }\end{array}$ & $\begin{array}{l}\text { Oryza } \\
\text { sativa, cv. } \\
\text { Nipponbare } \\
\text {; Hordeum } \\
\text { vulgare, } \\
\text { cv. Golden } \\
\text { Promise; } \\
\text { Triticum } \\
\text { aestivum, } \\
\text { cv. Gladius }\end{array}$ & $\begin{array}{l}\text { Agrobacterium } \\
\text { and } \\
\text { microprojectile } \\
\text { bombardment } \\
\text { mediated } \\
\text { transformed } \\
\text { lines }\end{array}$ & $\begin{array}{l}\text { Different } \\
\text { levels of } \\
\text { nitrogen }\end{array}$ & $\begin{array}{l}\text { Growth } \\
\text { parameters }\end{array}$ & $\begin{array}{l}\text { Transgenic, } \\
\text { transcriptomics, } \\
\text { metabolomics }\end{array}$ & $\begin{array}{l}\text { Alanine aminotransferase } \\
(H v A l a A T) \text {-overexpression } \\
\text { enhance NUE. }\end{array}$ & $\begin{array}{l}\text { Tiong et } \\
\text { al., } 2021\end{array}$ \\
\hline & Rice & $\begin{array}{l}\text { Oryza } \\
\text { sativa } L .\end{array}$ & $\begin{array}{l}\text { Indica and jap } \\
\text { onica rice } \\
\text { varieties }\end{array}$ & $\begin{array}{l}\text { Different } \\
\text { nitrogen } \\
\text { levels }\end{array}$ & Yield traits & $\begin{array}{l}\text { Genomics, } \\
\text { transcriptomics, } \\
\text { metabolomics, } \\
\text { and phenomics }\end{array}$ & $\begin{array}{l}\text { Quantitative trait locus } \\
\text { DULL NITROGEN } \\
\text { RESPONSE1 involved NUE } \\
\text { by auxin-mediated } \\
\text { signaling. }\end{array}$ & $\begin{array}{l}\text { Zhang et } \\
\text { al., } 2021\end{array}$ \\
\hline & Eggplant & $\begin{array}{l}\text { Solanum } \\
\text { melongena }\end{array}$ & Four genotypes & $\begin{array}{l}\text { Short- and } \\
\text { long-term } \\
\text { exposure to } \\
\text { low nitrogen }\end{array}$ & $\begin{array}{l}\text { Photosyntheti } \\
\text { c traits }\end{array}$ & Transcriptome & $\begin{array}{l}\text { High-NUE genotypes are } \\
\text { involved in the light- } \\
\text { harvesting complex and } \\
\text { receptor, a ferredoxin-- } \\
\text { NADP reductase, a catalase } \\
\text { and WRKY33. }\end{array}$ & $\begin{array}{l}\text { Mauceri } \\
\text { et al., } \\
2021\end{array}$ \\
\hline & $\begin{array}{l}\text { Pearl } \\
\text { millet }\end{array}$ & $\begin{array}{l}\text { Pennisetum } \\
\text { glaucum }(L \\
\text {.) } R . B r\end{array}$ & $\begin{array}{l}380 \text { diverse } \\
\text { pearl millet } \\
\text { lines }\end{array}$ & $\begin{array}{l}\text { Three } \\
\text { different N } \\
\text { levels- 0\%- } \\
\text { N0, } 50 \%- \\
\text { N50, } 100 \%- \\
\text { N100 }\end{array}$ & $\begin{array}{l}\text { Grain yield } \\
(\mathrm{GY})\end{array}$ & $\begin{array}{l}\text { Biochemical } \\
\text { and } \\
\text { performance } \\
\text { based on } \\
\text { phenotyping }\end{array}$ & $\begin{array}{l}\text { Identified } 25 \text { nitrogen } \\
\text { insensitive lines. }\end{array}$ & $\begin{array}{l}\text { Pujarula } \\
\text { et al., } \\
2021\end{array}$ \\
\hline
\end{tabular}

\section{resistance}




\begin{tabular}{|c|c|c|c|c|c|c|c|}
\hline $\begin{array}{l}\text { Oilseed } \\
\text { rape }\end{array}$ & $\begin{array}{l}\text { Brassica } \\
\text { napus } L .\end{array}$ & Two genotypes & $\begin{array}{l}\text { With or } \\
\text { without } \\
\text { nitrogen }\end{array}$ & $\begin{array}{l}\text { Sugar } \\
\text { concentrations }\end{array}$ & Transcriptome & $\begin{array}{l}\text { A vast number of genes are } \\
\text { associated with N-stress } \\
\text { response and high NUE. } \\
\text { These genes are involved in } \\
\text { phytohormone signaling, N } \\
\text { uptake and assimilation, } \\
\text { amino acid metabolism, } \\
\text { fatty acid biosynthesis, } \\
\text { photosynthesis, C } \\
\text { assimilation, starch } \\
\text { biosynthesis and } \\
\text { carbohydrate catabolism. }\end{array}$ & $\begin{array}{l}\text { Yang et } \\
\text { al., } 2020\end{array}$ \\
\hline Potato & $\begin{array}{l}\text { Solanum } \\
\text { tuberosum } \\
\text { L. }\end{array}$ & $\begin{array}{l}\text { Three potato } \\
\text { cultivars }\end{array}$ & $\begin{array}{l}\mathrm{N} \text {-complete } \\
\text { group (short } \\
\text { for } \mathrm{N} \text { ) and } \\
\mathrm{N} \text {-deficient } \\
\text { nutrient } \\
\text { group }\end{array}$ & -- & $\begin{array}{l}\text { Phenomics, } \\
\text { Transcriptomics } \\
\text { and biochemical }\end{array}$ & $\begin{array}{l}\text { Nitrogen deficiency } \\
\text { decreased the nitrate } \\
\text { reductase (NR), glutamine } \\
\text { synthetase (GS) and root } \\
\text { activity. }\end{array}$ & $\begin{array}{l}\text { Zhang et } \\
\text { al., } 2020\end{array}$ \\
\hline Brassica & $\begin{array}{l}\text { Brassica } \\
\text { napus }\end{array}$ & $\begin{array}{l}\text { Two } \\
\text { genotypes, D4- } \\
15 \text { and D2-1 } \\
\text { having } \\
\text { Contrasting } \\
\text { NUE }\end{array}$ & $\begin{array}{l}\text { Control and } \\
\text { nitrogen } \\
\text { starvation }\end{array}$ & Root traits & $\begin{array}{l}\text { Transcriptomics } \\
\text {, metabolomics, } \\
\text { and phenomics }\end{array}$ & $\begin{array}{l}\text { A nitrate transporter gene } \\
\text { BnaA06g04560D (NRT2.1) } \\
\text { and two vacuole nitrate } \\
\text { transporter } C L C \text { genes } \\
\text { (BnaA02g11800D and } \\
\text { BnaA02g28670D) were up- } \\
\text { regulated by N starvation in } \\
\text { N-efficient genotype (D4- } \\
\text { 15) }\end{array}$ & $\begin{array}{l}\text { Li et al., } \\
2020\end{array}$ \\
\hline $\begin{array}{l}\text { Bermuda } \\
\text { grass }\end{array}$ & $\begin{array}{l}\text { Cynodonda } \\
\text { ctylon L. }\end{array}$ & 290 genotypes & $\begin{array}{l}\text { High and } \\
\text { low nitrogen }\end{array}$ & Plant biomass & -- & -- & $\begin{array}{l}\text { Schneide } \\
\text { r-Canny } \\
\text { et al., } \\
2019\end{array}$ \\
\hline
\end{tabular}

These genes are involved

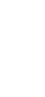

(1)




\begin{tabular}{|c|c|c|c|c|c|c|c|}
\hline Wheat & $\begin{array}{l}\text { Triticum } \\
\text { aestivum L. }\end{array}$ & 15 genotype & $\begin{array}{l}\text { Low and } \\
\text { optimum } \\
\text { nitrogen } \\
\text { levels }\end{array}$ & $\begin{array}{l}\text { Plant biomass } \\
\text { and grain per } \\
\text { pot }\end{array}$ & Phenotyping & -- & $\begin{array}{l}\text { Nguyen } \\
\text { et al., } \\
2019\end{array}$ \\
\hline Rice & $\begin{array}{l}\text { Oryza } \\
\text { sativa var. } \\
\text { indica }\end{array}$ & 15 genotype & $\begin{array}{l}\text { Low } \\
\text { nitrogen }\end{array}$ & $\begin{array}{l}\text { N-responsive } \\
\text { germination, } \\
\mathrm{O}_{2-} \\
\text { consumption, } \\
\text { seed urease } \\
\text { activities, root } \\
\text { growth, crop } \\
\text { duration, and } \\
\text { yield }\end{array}$ & $\begin{array}{l}\text { Performance- } \\
\text { based on } \\
\text { phenotyping and } \\
\text { biochemical }\end{array}$ & -- & $\begin{array}{l}\text { Sharma } \\
\text { et al., } \\
2018\end{array}$ \\
\hline Rice & $\begin{array}{l}\text { Oryza } \\
\text { sativa L. } \\
\text { subsp. japo } \\
\text { nica Kato }\end{array}$ & $\begin{array}{l}14 \text { indices of } \\
38 \text { varieties }\end{array}$ & $\begin{array}{l}\text { Three } \\
\text { different } \\
\text { nitrogen } \\
\text { levels }\end{array}$ & Relative yield & $\begin{array}{l}\text { Performance- } \\
\text { based on } \\
\text { phenotyping }\end{array}$ & -- & $\begin{array}{l}\text { Jai et al., } \\
2010\end{array}$ \\
\hline
\end{tabular}

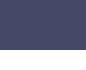

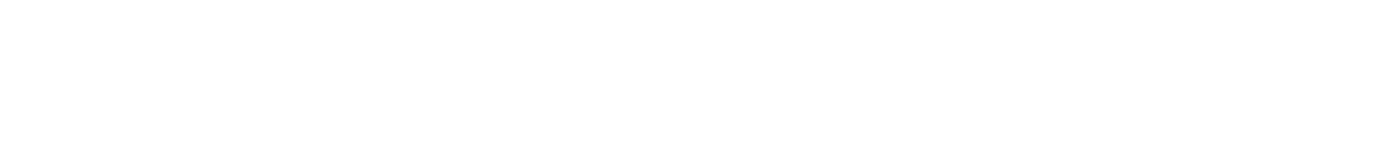

(1)

(2)


Above discussion clearly indicates systematic approaches are still lacking to understand WUE and NUE because of (1) the complexity of polygenic regulation, (2) both traits being highly influenced by environmental factors, (3) lack of investigations at the molecular level, and (4) lack of advancement of integrated approaches. Moreover, the molecular perspective in terms of the identification of desired genes, co-expression/interaction patterns, the influence of cis/trans-elements, and metabolic pathways of crop WUE and NUE was not addressed well. Remarkably, when executed in the real field trial, a large number of published trait-based experiments on controlled-environment conditions did not show consistency (Rebetzke et al., 2014). At present, automated robotics phenomic platforms have been used to quantify genotypes based on functional traits such as growth form, woodiness, leaf economic spectrum, WUE, NUE, as well as stress tolerance capacity. To explore the diverse gene bank resources for developing climate resilience crops, the evaluation of agronomic performance and stress-tolerant capacity under different climatic conditions along with crop behaviour, intra/inter-specific variability, and mutant performance evaluation is necessary. Therefore, to quantify the potentiality of genotypes, next-generation trait-based evaluation should be performed on field conditions. This will help us identify superior genotypes/accessions that are stable, abundant, and environmentally less sensitive (Martin et al., 2015). For identifying the desired genes and associated treats, the recent trend of the functional phenomic approach will be helpful (Braun et al., 2020). The availability of these resources will be accelerated by increasing systems biology approaches to understand the molecular mechanism of complex traits such as WUE and/or NUE (Figure 2). 


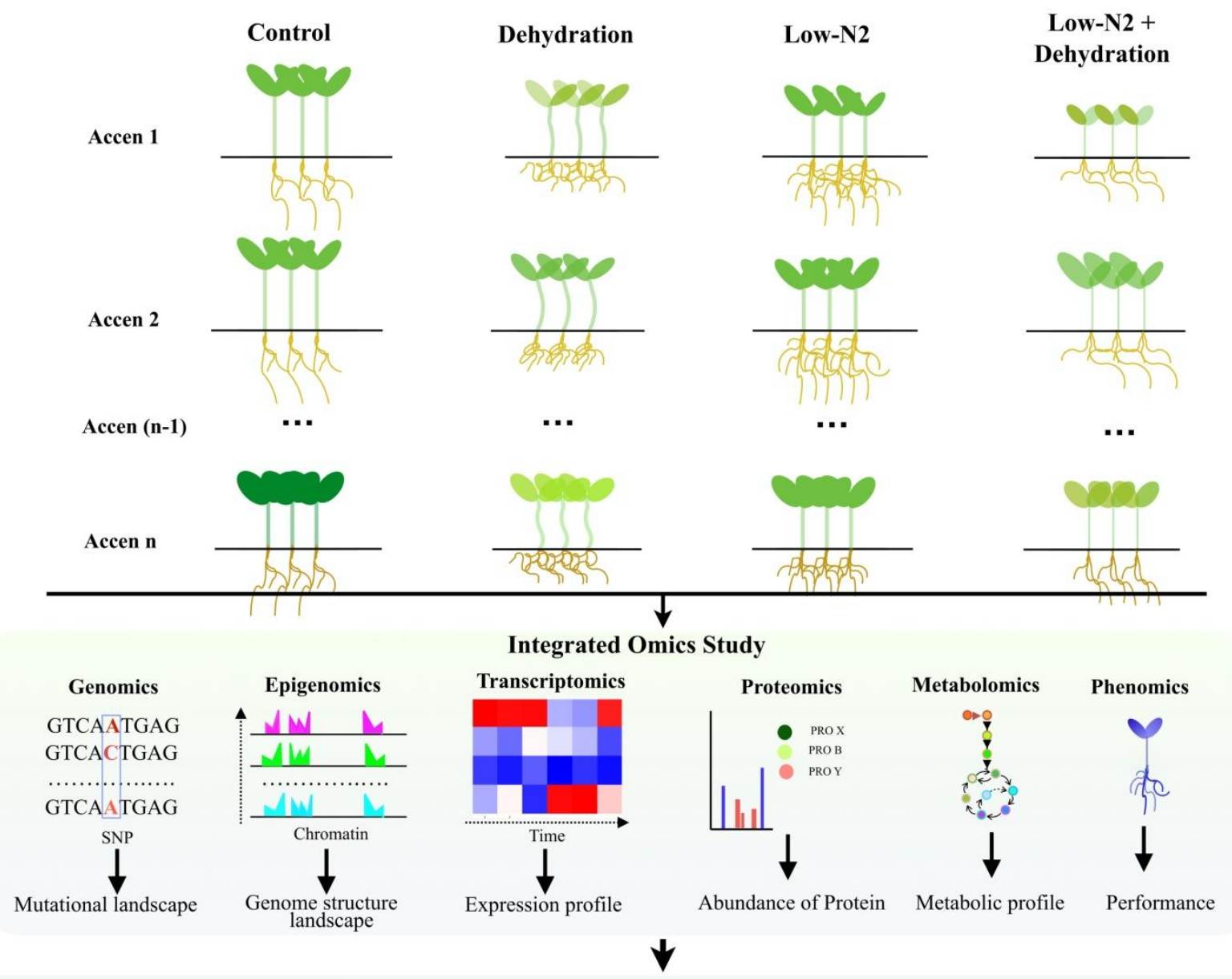

"Big-Data": Enrichment and Functional Network Analysis by Artificial Intelligence (AI)

(A) Gene Detection

\begin{tabular}{|c|c|}
\hline Gene & Biological Process \\
\hline G1 & N-metabolism process \\
\hline G2 & Oxidative metabolism \\
\hline G3 & Developmental process \\
\hline $\mathrm{G} 4$ & Response to drought \\
\hline G5 & Response to stress \\
\hline G6 & Aging \\
\hline G7 & Leaf senescence \\
\hline G8 & Nucleotide metabolism \\
\hline$\cdots$ & $\cdots$ \\
\hline Gn & Response to metal stress \\
\hline
\end{tabular}

(B) Interacting Network Analysis

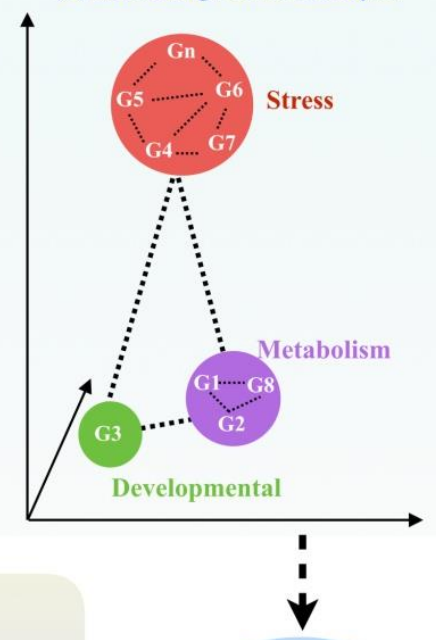

Modelling
Approaches

1

(C) Functional Phenomics

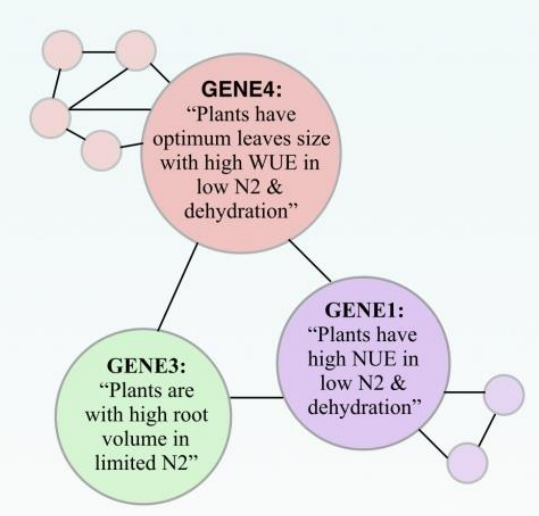

Guided Model

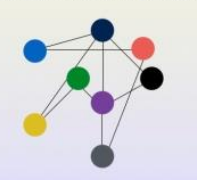

Understanding of complex traits with genetic background Understanding the contribution of rare alleles

Understanding of $G * E$ interactions

Easy to identify superior parental lines for promising breeding 
Figure 2: A comprehensive gene discovery strategy of water use efficiency (WUE) and nitrogen use efficiency (NUE) associated traits. Synthesized high-throughput multi-omic integration data will confirm the phenotypic behavior, mutational landmark (SNP), expression pattern, the abundance of protein, and metabolic signature with associated treatment. Different transcript, metabolic, protein-protein interaction libraries of different genotypes will be helpful for enrichment and functional network analysis. Previously reported different data-mining bioinformatics-based analysis, e.g., (a) NGS-based RNAseq study will be helpful to identify abundant genes, (b) multidimensional clustering and gene network analysis of proteomic study (2D gel electrophoresis and co-immunoprecipitation, etc.), and (c) functional phenomics that will help to predict and discrimination of the desired gene with the associated trait. The identification of a gene that is strongly associated with a particular phenotype, e.g., GENE 1 strongly associated with those plants that have high NUE in low $\mathrm{N}_{2}$ and dehydration conditions can be analyzed. Finally, big-data enrichment and functional network analysis by artificial intelligence (AI) will provide the opportunity to develop different types of models such as random models and guided models. The guided model will be more precious and accurate, which may develop as per our requirement, whereas a random model represents the overall analysis. Thus, an intelligently guided model will help in the understanding of complex traits with genetic background, $G^{*} E$ interactions patterns, developing ideotype breeding especially for underground traits, identify superior parental lines for promising breeding, improving smart farming.

\section{System biology and future sustainability}

To understand the genetic basis of trait variation, NGS-based genome-wide association studies (GWAS) are a powerful tool. This approach has been successfully applied to many important traits in different species, including yield-relevant traits, in crops. Sufficiently powered GWAS often identify tens to hundreds of loci containing hundreds of singlenucleotide polymorphisms (SNPs) associated with a trait of interest (McMullen et al., 2009). Once traits are correlated with specific biosynthetic/metabolic pathways and desired alleles have been identified, researchers can take up a broader understanding of crop biology to anticipate parental and allelic combinations that will discover superior agronomic traits. Although simply analyzing the genome sequences or genome-wide association study will become a "null hypothesis", we will never get the account of gene regulation from gene/protein sequences. To date, the identification of candidate genes detected by 
transcriptomics approaches and/or mapping is not available in most crops (Li et al., 2019). Furthermore, it is important to understand in-depth molecular mechanisms of their possible agronomic values. In addition to structural genomics, information on gene expression atlases, epigenome maps, proteome maps, and metabolome maps have been developed for a few crop species (Zogli et al., 2020). From the last ten years, plant molecular geneticists efficiently practice the most sophisticated toolkits for digging into valuable genetic resources that have been used such as advanced cloning strategies, genetic transformation methods, different gene-editing tools, co-immunoprecipitation, two-hybrid system, mass spectrometry, and datamining bioinformatics. In this context, the recent study published by Jia et al. (2020) in Nature Communications explains that KERNEL NUMBER PER ROW6 regulates maize grain yield, which is an outstanding example for us. Moreover, the information on the genetic architecture of important agronomical traits, their associated alleles, and the underlying molecular mechanism has remained elusive. Hence, to understand the complex biological phenomena, ongoing trends of "omic"-study will be helpful for crop improvement. For breeders/geneticists, transforming PGR into a bio-digital resource system is a prerequisite and helps us build up a database.

Germplasm is the source of novel genes. For global sustainability, selected promising genetic resources and genomic regions associated with the traits, integrated breeding approaches have been taken up. The classical paradigm of plant breeding, recurring cycles of crosses, and selections (MAS/MABC) have long been considered as a tedious method, whereas targeted genetic recombination has long been considered as a quicker method for the genetic transformation of the gene of interest from superior donors. Moreover, an array of reports suggested that plant tissue culture technology has shown considerable impact on the crop enhancement program. However, because of the lower frequency of transformation, in vitro recalcitrance, and different bio-safety rules, plant biotechnologists are unable to achieve the targets to date. The precise genetic engineering technology will allow the rapid transfer of advantageous traits between crops and their wild relatives in both directions. Furthermore, implementation of advanced breeding technologies including speed breeding dramatically accelerates crop development by facilitating fast generation cycles (Watson et al., 2018). Understanding the functional aspects, the conventional genetic methods-integrated strategy has the following advantages: (a) less tedious because of the multiplication of population is not required; (b) large plant sample size, mutants, and stable homozygous overexpressed plants are not required; (c) low-cost of mining of publicly available database resources makes the comparative study of omics data easier; (d) an integrated approach could identify and 
provide comprehensive interactions, co-expression, and metabolic/biosynthetic grid; and finally (e) integration of advanced artificial intelligence (AI) gives the sufficient multidimensional data. Thus, this integrated approach should turn out to be one of the most efficient systems for a high-resolution understanding of the complex genetic mechanisms in the near future. For crop improvement, the recent trends of genome editing toolkits have significant potentiality. As a plant geneticist/breeder, our aim is to focus on the exploration and strengthen the advanced strategies to harness the benefit of $\sim 7.4$ million gene bank germplasm material. Our present brief discussion will provide the advancement of germplasm assets that will meet the required sustainability in the near future.

Acknowledgment: The authors would like to thank the anonymous reviewer(s) for providing their valuable time, comments, and suggestions to improve the quality of this study for publication. The authors are grateful to Director, Central Sericultural Germplasm Resources Centre (CSGRC), Hosur, India, for the facilities provided to perform this research work.

Declaration of Funding: This research did not receive any specific funding.

Conflicts of interest: All the authors declare that there are no conflicts of interest in connection with this paper

\section{References:}

Arab MM, Marrano A, Abdollahi-Arpanahi R, Leslie CA, Cheng H, Neale DB, Vahdati K (2020) Combining phenotype, genotype, and environment to uncover genetic components underlying water use efficiency in Persian walnut. Journal of experimental botany; 71(3):1107-27.

Bailey-Serres J, Parker JE, Ainsworth EA, Oldroyd GE, Schroeder JIBailey-Serres, J., Parker, J.E., Ainsworth, E.A., Oldroyd, G.E. and Schroeder, J.I. (2019) Genetic strategies for improving crop yields. Nature, 575(7781), 109-118.

Braun, I.R., Yanarella, C.F. and Lawrence-Dill, C.J., (2020). Computing on Phenotypic Descriptions for Candidate Gene Discovery and Crop Improvement. Plant Phenomics, 2020, 1963251.

Connor R, Renata A, Ortigara C, Koncagül E, Uhlenbrook S, Lamizana-Diallo BM, Zadeh SM, Qadir M, Kjellén M, Sjödin J, Hendry S (2017) The united nations world water development report 2017. wastewater: the untapped resource. The United Nations World Water Development Report. 2017. 
De Souza AP, Wang Y, Orr DJ, Carmo-Silva E, Long SP (2020) Photosynthesis across African cassava germplasm is limited by Rubisco and mesophyll conductance at steady state, but by stomatal conductance in fluctuating light. New Phytologist; 225(6):2498-512.

Dharmappa PM, Doddaraju P, Malagondanahalli MV, Rangappa RB, Mallikarjuna NM, Rajendrareddy SH, Ramanjinappa R, Mavinahalli RP, Prasad TG, Udayakumar M, Sheshshayee SM (2019) Introgression of root and water use efficiency traits enhances water productivity: An evidence for physiological breeding in rice (Oryza sativa L.). Rice; 12(1):1-4.

Fagodiya RK, Kumar A, Kumari S, Medhi K, Shabnam AA (2020) Role of nitrogen and its agricultural management in changing environment. In Contaminants in Agriculture, 247270). Springer, Cham.

Fried HG, Narayanan S, Fallen B (2019) Evaluation of soybean [Glycine max (L.) Merr.] genotypes for yield, water use efficiency, and root traits. PloS one;14(2):e0212700.

Jamil M, Ali A, Akbar KF, Ghafoor A, Aziz AB, Napar SA, Mujeeb-Kazi A (2016) Relationship among water use efficiency, canopy temperature, chlorophyll content and spot blotch (Cochliobolus sativus) resistance in diverse wheat (Triticum aestivum L.) Germplasm. Pak. J. Bot; 48(3):993-8.

Janzen GM, Wang L, Hufford MB (2019) The extent of adaptive wild introgression in crops. New Phytologist. 221(3):1279-88.

Jia C, Wang F, Yuan J, Zhang Y, Zhao Z, Abulizi B, Wen X, Kang M, Tang F (2020) Screening and comprehensive evaluation of rice (Oryza sativa L. subsp. japonica Kato) germplasm resources for nitrogen efficiency in Xinjiang, China. Plant Genetic Resources;18(3):179-89.

Jia H, Li M, Li W, Liu L, Jian Y, Yang Z, Shen X, Ning Q, Du Y, Zhao R, Jackson D (2020). A serine/threonine protein kinase encoding gene KERNEL NUMBER PER ROW6 regulates maize grain yield. Nature communications,. 2020;11(1):1-1.

Kant S, Bi YM, Rothstein SJ (2011) Understanding plant response to nitrogen limitation for the improvement of crop nitrogen use efficiency. Journal of experimental Botany; 62(4):1499-509.

Kumar A, Medhi K, Fagodiya RK, Subrahmanyam G, Mondal R, Raja P, Malyan SK, Gupta DK, Gupta CK, Pathak H (2020) Molecular and ecological perspectives of nitrous oxide producing microbial communities in agro-ecosystems. Reviews in Environmental Science and Bio/Technology:1-34.

Langridge, P. and Waugh, R., (2019). Harnessing the potential of germplasm collections. Nature Genetics, 51(2), 200-201. 
Lavarenne J, Guyomarc'h S, Sallaud C, Gantet P, Lucas MLavarenne, J., Guyomarc'h, S., Sallaud, C., Gantet, P. and Lucas, M., (2018.) The spring of systems biology-driven breeding. Trends in plant science, 23(8), 706-720.

Li M, Xu J, Gao Z, Tian H, Gao Y, Kariman K (2020) Genetically modified crops are superior in their nitrogen use efficiency-A meta-analysis of three major cereals. Scientific reports; 10(1):1-9.

Li Q, Ding G, Yang N, White PJ, Ye X, Cai H, Lu J, Shi L, Xu F (2020) Comparative genome and transcriptome analysis unravels key factors of nitrogen use efficiency in Brassica napus L. Plant, cell \& environment; 43(3):712-31.

Li T, Wang YH, Liu JX, Feng K, Xu ZS, Xiong AS (2019). Advances in genomic, transcriptomic, proteomic, and metabolomic approaches to study biotic stress in fruit crops. Critical reviews in biotechnology, 39(5), 680-692.

Lopez MA, Xavier A, Rainey KM (2019) Phenotypic variation and genetic architecture for photosynthesis and water use efficiency in soybean (Glycine max L. Merr). Frontiers in plant science; $10: 680$.

Martin, A.R,. and Isaac, M.E., (2015). Plant functional traits in agroecosystems: a blueprint for research. Journal of Applied Ecology, 52(6), 1425-1435.

Mascher M, Schreiber M, Scholz U, Graner A, Reif JC, Stein N (2019.) Genebank genomics bridges the gap between the conservation of crop diversity and plant breeding. Nature genetics, 51(7), 1076-1081.

Mauceri A, Rosa Abenavoli M, Toppino L, Panda S, Mercati F, MiyassaAci M, Aharoni A, Sunseri F, Rotino GL, Lupini A (2021) Transcriptomic insights on molecular regulation of Solanum melongena L. N-Use Efficiency. Journal of Experimental Botany.

McMullen MD, Kresovich S, Villeda HS, Bradbury P, Li H, Sun Q, Flint-Garcia S, Thornsberry J, Acharya C, Bottoms C, Brown P (2009) . Genetic properties of the maize nested association mapping population. Science. 2009 Aug 7;325(5941):737-40.

Meena RP, Karnam V, Sendhil R, Sharma RK, Tripathi SC, Singh GP (2019) Identification of water use efficient wheat genotypes with high yield for regions of depleting water resources in India. Agricultural Water Management; 223:105709.

Nguyen GN, Maharjan P, Maphosa L, Vakani J, Thoday-Kennedy E, Kant S (2019) A robust automated image-based phenotyping method for rapid vegetative screening of wheat germplasm for nitrogen use efficiency. Frontiers in plant science;10:1372.

Pignon C, Fernandes S, Valluru R, Bandillo N, Lozano R, Buckler ES, Gore MA, Long SP, Brown PJ, Leakey A (2021) Phenotyping stomatal closure by thermal imaging for GWAS and TWAS of water use efficiency-related genes. bioRxiv. Jan 1. 
Pignon CP, Leakey AD, Long SP, Kromdijk J (2021) Drivers of natural variation in wateruse efficiency under fluctuating light are promising targets for improvement in sorghum. Frontiers in plant science; 12.

Pujarula V, Pusuluri M, Bollam S, Das RR, Ratnala R, Adapala G, Thuraga V, Rathore A, Srivastava RK, Gupta R (2021) Genetic variation for nitrogen use efficiency traits in global diversity panel and parents of mapping populations in pearl millet. Frontiers in Plant Science; $12: 73$.

Pujarula V, Pusuluri M, Bollam S, Das RR, Ratnala R, Adapala G, Thuraga V, Rathore A, Srivastava RK, Gupta R (2021) Genetic variation for nitrogen use efficiency traits in global diversity panel and parents of mapping populations in pearl millet. Frontiers in Plant Science $; 12: 73$.

Raju BR, Mohankumar MV, Sumanth KK, Rajanna MP, Udayakumar M, Prasad TG, Sheshshayee MS (2016) Discovery of QTLs for water mining and water use efficiency traits in rice under water-limited condition through association mapping. Molecular Breeding; 36(3):35.

Raun WR, Johnson GV (1999) Improving nitrogen use efficiency for cereal production. Agronomy journal; 91(3):357-63.

Rebetzke GJ, Fischer RT, Van Herwaarden AF, Bonnett DG, Chenu K, Rattey AR, Fettell NA (2014). Plot size matters: interference from intergenotypic competition in plant phenotyping studies. Functional Plant Biology, 41(2), 107-118.

Schneider-Canny R, Chekhovskiy K, Munoz P, Kwon S, Saha M (2019) Characterization of bermudagrass (Cynodondactylon L.) germplasm for nitrogen use efficiency. Euphytica; 215(3):40.

Sharma N, Sinha VB, Gupta N, Rajpal S, Kuchi S, Sitaramam V, Parsad R, Raghuram N (2018) Phenotyping for nitrogen use efficiency: rice genotypes differ in N-responsive germination, oxygen consumption, seed urease activities, root growth, crop duration, and yield at low N. Frontiers in plant science; 9:1452.

St Aime R, Rhodes G, Jones M, Campbell BT, Narayanan S (2021) Evaluation of root traits and water use efficiency of different cotton genotypes in the presence or absence of a soil-hardpan. The Crop Journal.

Tiong J, Sharma N, Sampath R, MacKenzie N, Watanabe S, Metot C, Lu Z, Skinner W, Lu Y, Kridl J, Baumann U (2021) Improving nitrogen use efficiency through overexpression of alanine aminotransferase in rice, wheat, and barley. Frontiers in plant science;12. 
Tracy SR, Nagel KA, Postma JA, Fassbender H, Wasson A, Watt M (2020). Crop improvement from phenotyping roots: Highlights reveal expanding opportunities. Trends in plant science, 25(1), 105-118.

Varshney RK, Sinha P, Singh VK, Kumar A, Zhang Q, Bennetzen JL (2020.) 5Gs for crop genetic improvement. Current Opinion in Plant Biology. 56, 190-196

Watson A, Ghosh S, Williams MJ, Cuddy WS, Simmonds J, Rey MD, Hatta MA, Hinchliffe A, Steed A, Reynolds D, Adamski NM (2018). Speed breeding is a powerful tool to accelerate crop research and breeding. Nature plants, 4(1),23-29.

Yang N, Li S, Wang S, Li Q, Xu F, Shi L, Wang C, Ye X, Cai H, Ding G (2020) Dynamic transcriptome analysis indicates extensive and discrepant transcriptomic reprogramming of two rapeseed genotypes with contrasting NUE in response to nitrogen deficiency. Plant and Soil; 456(1):369-90.

Zhang J, Wang Y, Zhao Y, Zhang Y, Zhang J, Ma H, Han Y (2020) Transcriptome analysis reveals Nitrogen deficiency induced alterations in leaf and root of three cultivars of potato (Solanum tuberosum L.). PloS one;15(10):e0240662.

Zogli P, Pingault L, Grover S, Louis J (2020.) Ento(o)mics: the intersection of 'omic' approaches to decipher plant defense against sap-sucking insect pests. Current Opinion in Plant Biology, 56, 153-161. 